\title{
Social media addiction: technological déjà vu
}

Brittany I Davidson doctoral researcher in information systems ${ }^{1}$

David A Ellis lecturer in computational social science ${ }^{2}$

${ }^{1}$ Information, Decisions, and Operations (IDO) Division, School of Management, University of Bath, Bath BA2 7AY, UK

${ }^{2}$ Department of Psychology, Lancaster University, Lancaster LA1 4YW, UK

Zendle and Bowen-Jones are correct when they point towards methodological shortcomings in research that aims to understand the impact of social media [1]. However, these observations apply to related research, which considers the impact of technology use more generally. Research typically involves asking people to consider their personal experience with a technology [2]. This reflects a general shift away from behavioral measurement in psychology $[3,4]$. In a recent article, we tested the predictive ability of popular assessment inventories used to quantify smartphone usage. Unsurprisingly, these scales did not align well with even the most basic measures of objective behavior, including those associated with compulsive use (e.g., rapid checking) [1,5]. It remains unclear exactly what these assessment tools are measuring, however, survey items are conceptually very similar to depression and anxiety scales. This alone may explain small negative associations between technology use and mood.

In addition, narratives surrounding the mass adoption of new technologies are recurrently negative. In response, researchers in the area might want to start asking themselves exactly why or how the use of social communication technology would cause harm and develop measures accordingly. Social media is certainly not the first technology to be associated with potentially 'addictive' or negative societal impacts [6,7], nor will it be the last. Moral panics concerning new technology (e.g., the printing press, the telephone, microwaves, the internet, social media) are, historically speaking, either overblown or demonstrably false.

Understanding the impact of technology on people and society remains crucial, but the field might also want to consider what drives 'technophobia' in the first place?

1. Ellis DA, Davidson BI, Shaw H, Geyer K. Do smartphone usage scales predict behavior? Int J Human-Computer Stud Spec Issue Hum Accuracy. 2019;

2. Ellis DA. Are smartphones really that bad? Improving the psychological measurement of technology-related behaviors. Comput Human Behav. 2019;97:60-6.

3. Doliński D. Is Psychology Still a Science of Behaviour? Soc Psychol Bull. 2018;

4. Sassenberg K, Ditrich L. Research in Social Psychology Changed Between 2011 and 2016: Larger Sample Sizes, More Self-Report Measures, and More Online Studies. Adv Methods Pract Psychol Sci. 2019;2515245919. 
5. Andrews S, Ellis DA, Shaw H, Piwek L. Beyond self-report: Tools to compare estimated and real-world smartphone use. PLoS One. 2015;10(10):1-9.

6. Parker S. Science Discoveries: Alexander Graham Bell. Philadelphia, USA: Chelsea House Publishers; 1995.

7. Edgerton D. Technophobis then and now. Nature. 1995;376(6542):653-4. 